\title{
The contribution of color to attention capture effects during search for onset targets
}

\author{
Florian Goller $^{1,2} \cdot$ Thomas Ditye $^{1} \cdot$ Ulrich Ansorge $^{1}$
}

Published online: 7 January 2016

(C) The Psychonomic Society, Inc. 2016

\begin{abstract}
The literature on top-down contingent capture is concerned with the question of what constitutes a search set. Is it restricted to single stimulus properties such as color or onsets, or can such sets be more complex? In nine experiments $(N=140)$, we tested whether cueing effects during search for onset targets were affected by cue color. According to the classic theory of contingent capture (Folk, Remington, \& Johnston, Journal of Experimental Psychology: Human Perception and Performance, 18, 1030-1044, 1992), during search for onset targets, cues capture attention on the basis of a match between the cue's onset and top-down control settings directed to the target onsets. However, such cueing effects were based on cues of a color similar to the target color. Therefore, matches of the cue color to the target color could have contributed to the effects. Indeed, here we found cueing effects when the cues and targets were of the same color, but not when they were of different colors (Exps. 1a, 1b, 4a, and 4b). In addition, same-color cueing effects were stronger than different-color cueing effects (Exps. 2a, 2b, 3a, 3b, and the white-target conditions of Exp. 5). In Experiment 5, we also identified efficient search for only one target color as a critical prerequisite for the differences between cueing by color-similar and -dissimilar onset cues. We conclude with a discussion of the contributions of cue-to-set color matches, deallocation of attention, and intertrial priming to what appear to be top-down contingent-capture effects based on abrupt onsets.
\end{abstract}

Florian Goller

florian.goller@univie.ac.at

1 Department of Psychology, University of Vienna, Vienna, Austria

2 Faculty of Psychology, University of Vienna, Liebiggasse 5, 1010 Wien, Austria
Keywords Cueing · Attention capture · Onset · Color · Contingent capture

At any time, humans perceive only some of the available visual information and ignore the rest. This selectivity is called visual attention and can be both stimulus-driven and topdown-dependent (Bundesen, Habekost, \& Kyllingsbæk, 2005; Wolfe, 1994). Strong evidence for top-down attentional control comes from contingent-capture effects, indicating that the capture of attention is contingent on attentional control settings (Folk, Remington, \& Johnston, 1992). For example, when looking for a lemon, observers might set their attentional control settings so as to find yellow (or yellower) objects in the environment (see Becker, Folk, \& Remington, 2010).

Originally, Folk et al. (1992) argued for the existence of two types of top-down control settings: settings for color (or static features) and settings for abrupt onsets (or dynamic features). This proposal was later revised when it became clear that control settings can even be selectively directed to only one specific color (Folk \& Remington, 1998). In the present study, we investigated the contribution of control settings for a specific color in cases that originally had been attributed to contingent capture by abrupt onsets. This is necessary because both color and onsets could have contributed to top-down contingent-capture effects for abrupt-onset stimuli, as we will explain next.

Folk et al. (1992) instructed participants to search for two different types of targets. In one block, participants searched for a white abrupt-onset target, and in the other block, they searched for a red color target presented together with white distractors. Prior to the target, two types of cues were presented: white onset cues shown in isolation, or red color cues shown together with white nonsingletons. Importantly, both types of cues were presented at either the same position as the 
target (valid condition) or at a different position than the target (invalid condition) and were not informative about the target position. In line with top-down contingent attentional capture, a selective cueing effect was found: Reaction times (RTs) were faster in valid than in invalid conditions, but only if the cue matched the top-down settings for the targets. If participants searched for a white abrupt-onset target, a white onset cue resulted in a cueing effect, but a red color cue did not; if participants searched for a red color target, a red color cue triggered a cueing effect, but a white onset cue did not.

Originally, Folk et al. (1992) assumed that participants used static control settings to search for the color targets. As a consequence, any pop-out color stimulus matching these control settings should capture attention. These authors also argued that participants used dynamic control settings to search for the onset targets, and that therefore any onset stimulus would match this control setting and capture attention. However, Folk and Remington (1998) revised these views when they observed that participants were able to ignore green color cues when searching for red color targets, and to ignore red color cues when searching for green color targets. In light of these findings, Folk and Remington argued that their participants set up different static control settings directed to just a specific color. This finding implies that the properties of the control settings depend on the task at hand.

Importantly, Folk and Remington (1998) did not revise their view on the mechanisms responsible for the cueing effects in the target-onset conditions, although the use of different color control settings could also explain these effects. In Folk et al. (1992), the onset cues were white and the color cues were red. Therefore, a control setting for white in the onsettarget conditions would have led to the same results as a control setting for abrupt onsets: capture by white and no capture by red color cues. In the present study we tested this possibility, because two findings have suggested that the top-down contingent capture by onset cues might be explained by search for colors alone.

First, search for onset targets seems to depend on target color: With red abrupt-onset targets, participants showed a stronger cueing effect with reddish than with greenish abrupt-onset cues. With green onset targets, the opposite was found (Ansorge \& Heumann, 2003, 2004; Ludwig \& Gilchrist, 2002; see also Anderson \& Folk, 2010, for similar findings with color singleton targets). This should not be if search were for abrupt onsets only. However, there was a residual cueing effect even with the color-dissimilar cues in most of these conditions. With the present study we aimed to extend these findings and to determine the time course of this cueing effect: By analyzing RTs in quintiles, we wanted to determine at which point in time the cueing effects for more and less color-similar onset cues start to differ from one another. Possibly attention is captured by both target-colorsimilar and-dissimilar cues, but participants are better at deallocating their attention away from dissimilar than from similar cues (Theeuwes, Atchley, \& Kramer, 2000). If deallocation plays a role, we would expect to find larger cueing effects for color-dissimilar cues among the faster responses that would decrease as RTs increased. If, however, dissimilar cues do not capture attention in any way, we would expect to find no cueing effects by color-dissimilar cues from the fastest RT quintile onward. Admittedly, deallocation could also take place prior to the fastest RTs, rendering null cueing effects of the dissimilar cues in the first RT quintile not entirely conclusive. Yet, theoretically, the quintile analysis could at least yield a pattern predicted by the deallocation account.

A second line of research suggests a role for top-down contingent capture by color in search for onset targets: Using black abrupt-onset targets, Fuchs, Theeuwes, and Ansorge (2013) showed a selective cueing effect with black abruptonset cues, and no cueing effect with white ones. This pattern reversed during search for white onset targets. However, Fuchs et al. used a detection task that was not perfectly sensitive to attentional capture effects. In light of these considerations and findings, we tested whether the contingent-capture effect of onset cues in a discrimination task (see Folk et al., 1992) is facilitated by an additional match between cue color and a target-directed top-down setting, or whether the cueing effects of onset cues can even be entirely explained by the degree to which these cues match the attentional control settings for colors. Furthermore, we set out to test how contingent attention capture by onset stimuli is boosted, or even entirely explained, by contingent capture by color.

\section{Experiment 1}

In Experiment 1, participants searched for white abrupt-onset targets. In Experiment 1a, either a white abrupt-onset cue (presented in isolation) or a white color cue (presented together with three red nonsingletons) preceded the target. If participants set their control settings for abrupt onsets, white onset cues should lead to a cueing effect, whereas white color cues should not. In contrast, if participants set their control settings for color, both of the cues should lead to cueing effects.

This would also be predicted if participants search for any kind of pop-out target (Bacon \& Egeth, 1994; Leber \& Egeth, 2006), or if pop-out features attract attention in a stimulusdriven way (Theeuwes, 2010). We addressed these possibilities in Experiment 1b, in which participants also searched for a white abrupt-onset target, but now this target was preceded by a white color cue (presented together with red nonsingletons) or a red color cue (presented together with white nonsingletons). If all pop-out stimuli attract attention, regardless of their color similarity to the searched-for targets, the white and the red color cues should both lead to cueing effects in Experiment 1b. However, if control settings for 
colors were at work, we would expect the white but not the red color cues to elicit a cueing effect. Finally, if control settings were directed to onsets, no cueing effect would be expected in Experiment 1b, because both of the cues were color cues.

\section{Method}

Participants Sixteen psychology students at the University of Vienna participated in Experiments 1a (ten female, six male; $M_{\mathrm{Age}}=22.89$ years, $\left.S D_{\mathrm{Age}}=2.86\right)$ and $1 \mathrm{~b}(11$ female, five male; $M_{\mathrm{Age}}=22.33$ years, $S D_{\mathrm{Age}}=2.13$ ), respectively. Here and in the following experiments, each student received partial course credit, had normal or corrected-to-normal visual acuity and normal color vision, and participated in only one of all nine experiments. Prior to all experiments, informed consent was obtained from all participants. In Experiments $1 \mathrm{a}$ and $1 \mathrm{~b}$, one participant was excluded because of an error rate exceeding $20 \%$.

Apparatus Stimuli were presented on a 19-in. LCD monitor (Acer B 193) with an aspect ratio of 4:3 and a resolution of 1 , $024 \times 768$ pixels at a vertical refresh rate of $75 \mathrm{~Hz}$. Viewing distance was $57 \mathrm{~cm}$, and participants' heads were supported by a chin and forehead rest. Manual responses were recorded as keypresses of the left and right index fingers on a standard keyboard. The experiment was controlled using MATLAB 7.7.0 (MathWorks Inc., Natick, MA, USA) and the Psychophysics Toolbox (Brainard, 1997; Pelli, 1997).

Stimuli All stimuli were presented against a black (CIE Lab coordinates: $\left.0.8 \mathrm{~cd} / \mathrm{m}^{2} ; 0.1 /-0.1\right)$ background. Four gray $\left(75.5 \mathrm{~cd} / \mathrm{m}^{2} ;-5.3 /-18.8\right)$ square-shaped outline placeholders $\left(1.5^{\circ} \times 1.5^{\circ}\right)$ were presented at the corners of an imaginary diamond around a central gray fixation point $\left(0.5^{\circ}\right)$. The center-to-center distance between the fixation point and the placeholders was $5.0^{\circ}$. On each trial, a white $\left(137.7 \mathrm{~cd} / \mathrm{m}^{2}\right.$; 7.8/-28.4) onset target was shown in the center of one of the placeholders. This target was equally likely an " $\mathrm{X}$ " or an "=" $\left(1.33^{\circ} \times 1.33^{\circ}\right)$. In Experiment 1a, one half of all cues (four filled dots with diameters of $0.25^{\circ}$ ) were white color cues centered around one placeholder and presented together with red nonsingletons $\left(74.2 \mathrm{~cd} / \mathrm{m}^{2} ; 94.4 / 98.3\right)$ centered around the other placeholders. The other half of all cues were white onset cues presented alone. In Experiment 1b, instead of the white onset cues, red color cues (presented together with white nonsingletons) were used (see also Fig. 1).

Procedure Figure 1 illustrates the sequence of events in a trial. After fixation $(600 \mathrm{~ms})$, the cueing display was presented for $50 \mathrm{~ms}$, followed by another fixation display for $100 \mathrm{~ms}$. Next, the target was shown for $50 \mathrm{~ms}$ on top of the fixation display. After the offset of the target, a blank screen was presented until participants reported the target's identity (" $X$ " or
“=”) via a keypress. Stimulus-to-response mappings were constant within but counterbalanced across participants. RTs were measured to the nearest millisecond as the time intervals between target onset and keypress. Release of the response key started the next trial. Participants were instructed to answer as quickly and accurately as possible. If no keypress was registered within $1,550 \mathrm{~ms}$, participants received onscreen feedback ("react faster!"), and the trial counted as an error.

From trial to trial, cue positions, target positions, and target identities varied randomly, but across trials, both cues and targets were counterbalanced across all four positions. The cue and target positions were $25 \%$ valid, $75 \%$ invalid, and uncorrelated across trials. Invalid cues appeared randomly but equally often at each of the three remaining positions, other than that of the target. The cues were not informative about the target's identity. Each of the two possible target shapes was presented equally often. The different conditions were presented in a pseudorandomized order.

\section{Results}

RTs of Experiment 1 Error trials and all trials with an RT below $150 \mathrm{~ms}$ were removed $(4.97 \%)$. The RTs for each participant and variable combination were sorted in ascending order and into equally sized quintiles: The fastest $20 \%$ of all RTs were sorted into Quintile 1, the slowest $20 \%$ of all RTs in Quintile 5, and the other RTs binned accordingly in between. We computed cueing effects as the differences of the mean correct RTs in invalid trials minus the mean correct RTs in valid trials, separately for each participant as well as for each step of the variable cue type (with varying steps; here, white onset cue vs. white color cue in Exp. 1a, and white color cue vs. red color cue in Exp. 1b) and for each quintile. Unless otherwise stated, all cueing effects are significantly different from zero (all $p \mathrm{~s}<.05$ ). Throughout this article, all results were processed in a corresponding way and further analyzed by a repeated measurements analysis of variance (ANOVA), with cue type and quintile as within-subjects variables. Where appropriate, degrees of freedom were adjusted by the Greenhouse-Geisser correction. For better transparency, the uncorrected degrees of freedom are reported. All reported pairwise comparisons were Bonferroni-corrected, meaning that the critical $p$ value of significance was multiplied by the number of comparisons.

RTs of Experiment 1a White color cues matched a potential top-down set for color, whereas white onset cues would have matched top-down sets for onsets and color. The analysis showed a main effect of quintile, $F(4,56)=8.34, p<.001$, indicating that the cueing effects were different between quintiles $(26,26,27,31$, and $48 \mathrm{~ms}$, respectively, from the 1 st to the 5 th quintile). Pairwise comparisons revealed that the cueing effect in the 5 th quintile was significantly larger than those 

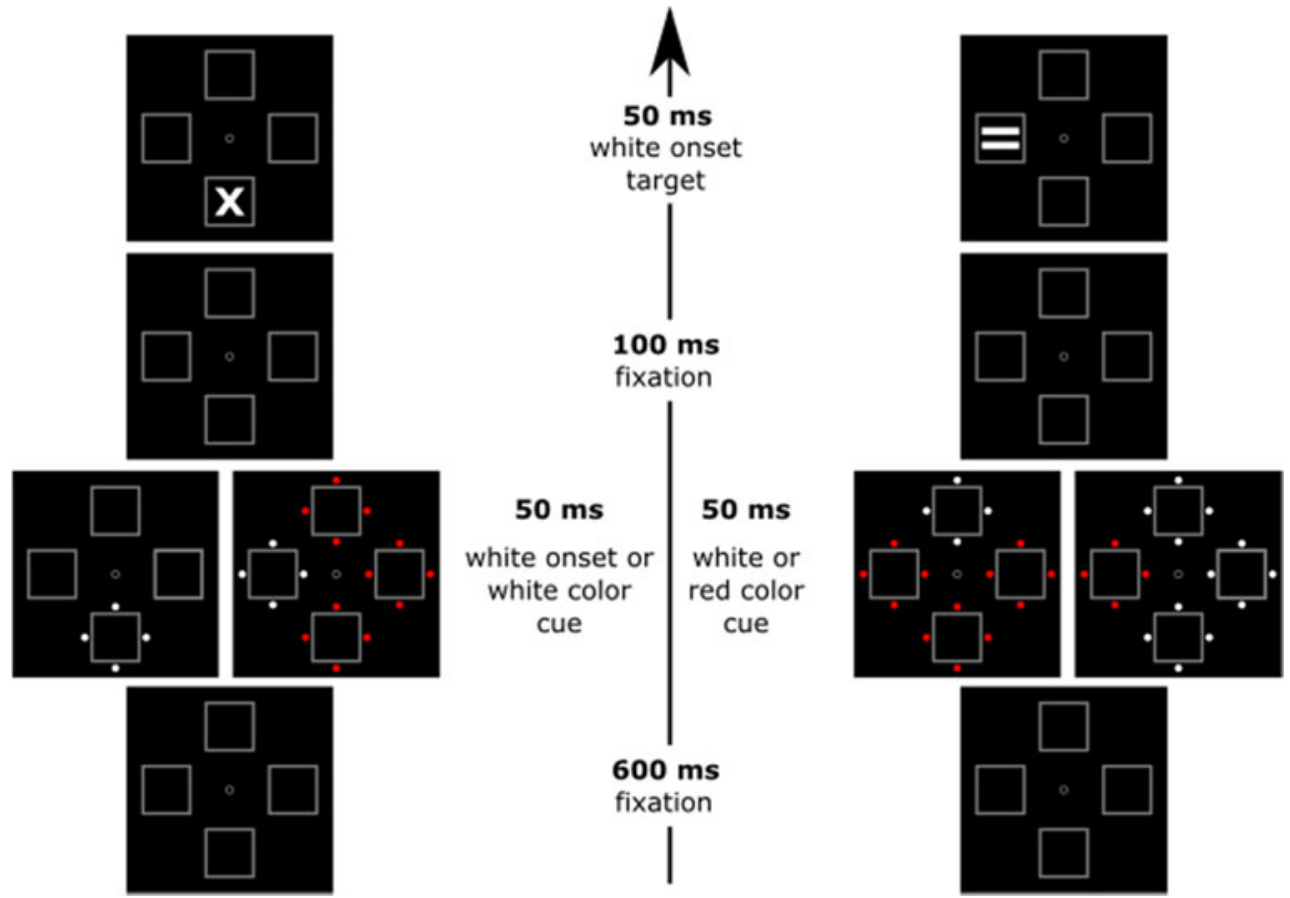

Fig. 1 Example sequence of Experiment 1a (left), and an example sequence of Experiment $1 \mathrm{~b}$ (right), both with time flowing from bottom to top. First, a fixation display was shown, followed by a cue display, another fixation display, and finally a target display.

in all other quintiles (all $p \mathrm{~s}<.011$ ). No other differences between the quintiles and no main effect of cue type were found. Figure 2 (upper panel) shows the cueing effects as a function of quintile and cue type. No other effects were found, all $F \mathrm{~s}<1.91$, all $p \mathrm{~s}>.121$.

Error rates (ERs) of Experiment 1a The arcsinetransformed ERs were subjected to a repeated measurements ANOVA, with the variables validity (valid, invalid) and cue type (white color cue, white onset cue). The results showed no significant effects, all $F_{\mathrm{s}}<1.05$, all $p \mathrm{~s}>.322$.

RTs of Experiment 1b The data were handled as in Experiment 1a, which led to the exclusion of $5.03 \%$ of all trials. White color cues matched a potential top-down set for colors, whereas red color cues did not match any set, be it for color or onsets. The analysis showed a main effect of cue type, $F(1,14)=137.08, p<.001$. The cueing effect was selectively present for white color cues (32 ms), $t(14)=10.14, p<.001$, but inverted for red color cues $(-11 \mathrm{~ms}), t(14)=-4.34$, $p<.001$. No other effects were found, all $F_{\mathrm{S}}<0.67$, all $p$ s $>$.617. The lower panel of Fig. 2 illustrates these cueing effects.

ERs of Experiment 1b The analysis of the ERs was analogous to that in Experiment 1a and yielded no significant results, all $F_{\mathrm{S}}<2.54$, all $p \mathrm{~s}>.134$.
In Experiment 1a, the white color cues and the white onset cues were used equally often, and in Experiment $1 \mathrm{~b}$, the white color cues and the red color cues were used equally often. The figure is not drawn to scale

\section{Discussion}

In both experiments, participants searched for white onset targets. In Experiment 1a, the cueing effects of white onset and white color cues were of the same size, although only the white onset cues would have matched a control setting for onsets. These results can be explained by control settings for target color, in which case top-down contingent attention capture of both cues would be predicted.

The results of Experiment 1a could also be explained as reflecting capture by pop-out. However, in Experiment 1b, the cueing effect was restricted to the white color cue. With the red color cue, the cueing effect was even inverted, although this cue was also a pop-out stimulus. Thus, the presence of a match of the cue to the set for target colors seemed to be both sufficient (Exp. 1a) and necessary (Exp. 1b) for a standard cueing effect of the onset cues.

Yet the following caveats should be taken into consideration. The inversion of the cueing effect in Experiment $1 \mathrm{~b}$ might indicate active suppression of the red color cue, inhibiting target processing at the position of this cue (cf. Lamy, Leber, \& Egeth, 2004; but see Carmel \& Lamy, 2014). Alternatively, in Experiment 1b's red color cue conditions, attention could have been deployed to all three white nonsingletons in the cueing display, or randomly to one of these nonsingletons at a time. This in turn could have led to RT costs if the target was shown at the actually least attended 


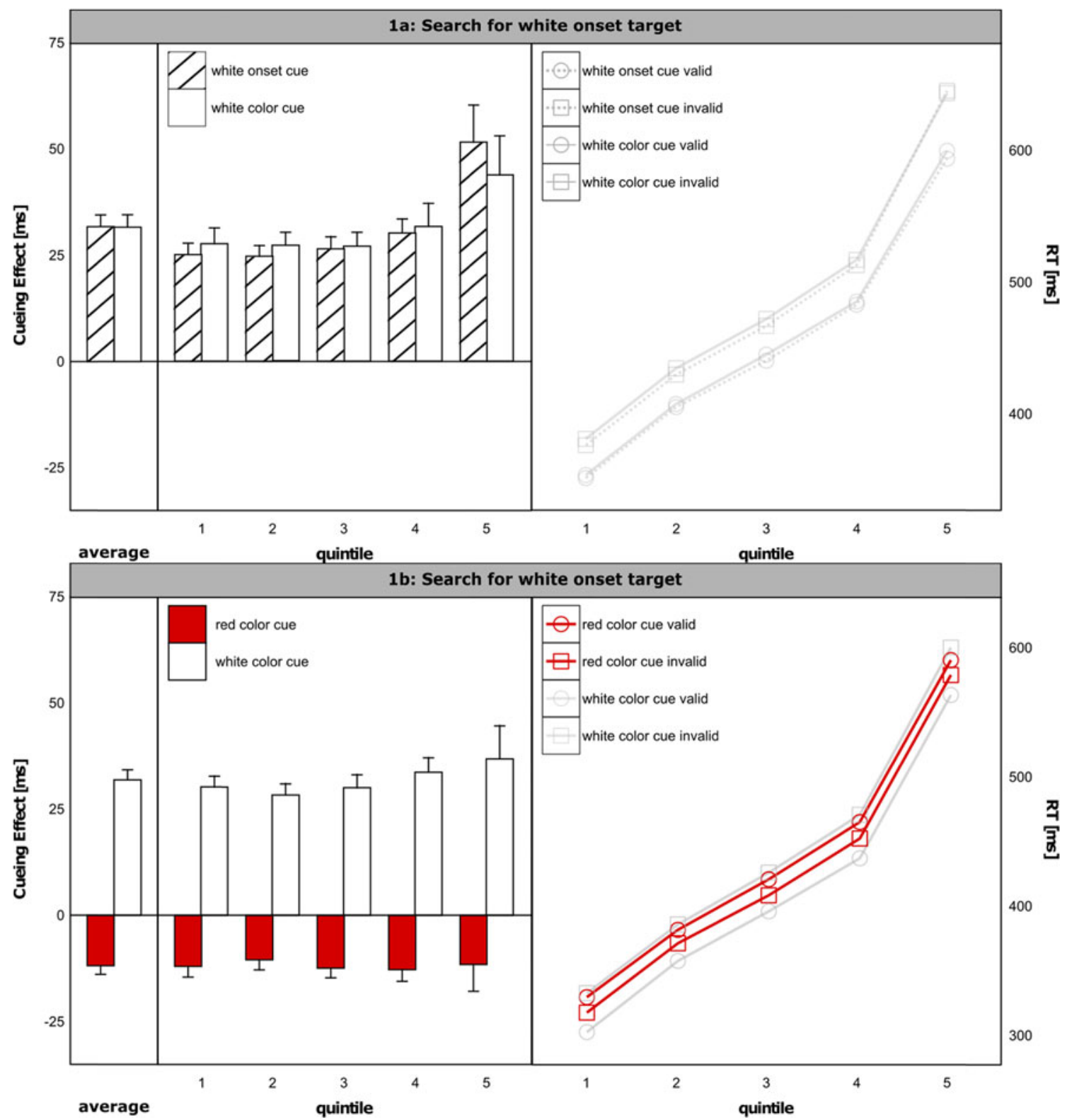

Fig. 2 From left to right: Cueing effects (invalid RT minus valid RT) as a function of cue type, averaged across quintiles; cueing effects as a function of cue type, split for the RT quintiles; and RTs as a function of

cue validity $($ circles $=$ valid, squares $=$ invalid $)$, cue type, and quintile $(x$ axis). The top panel depicts the results of Experiment 1a, and the bottom panel those of Experiment $1 \mathrm{~b}$

position of the red nonmatching cue. Whatever the exact principle, these results support an interpretation in terms of a cueing effect that was contingent on a top-down control setting for the target's white color.

Another caveat is that a search setting for chromaticity and a search setting for luminance transients could both explain our results, because the white stimuli always were the highestluminance transients in the display. If participants searched for luminance transients to find the white targets, a white onset cue and white color cue would have matched such a control setting in Experiment 1a. In the same manner, more attention capture by the white color cues in Experiment $1 \mathrm{~b}$ could be explained. At variance with this explanation, a top-down setting for luminance transients would have predicted more capture by white onset cues than by white color cues in Experiment 1a, because the white color cues would have suffered from a lower difference by which the cue's luminance stood out among the weaker but still luminant red nonsingletons. The issue whether sets for chromaticity or luminance accounted for the findings cannot ultimately be decided on the basis of the present results alone, and will be addressed in Experiments 2b, 3, 4, and 5.

A third caveat concerns the utility of a top-down setting for color as a means to ignore the red color cues in Experiment $1 \mathrm{~b}$. 
Maybe the participants in Experiment $1 \mathrm{~b}$ selected attentional control settings for the target's white color because this allowed them to both attend to the targets and ignore $50 \%$ of the uninformative cues - namely, the red color cues. Note that this incentive was also provided in most other contingent-capture experiments (e.g., Folk et al., 1992; Folk \& Remington, 1998).

In any case, the difference between the cueing effects of matching white color cues and of nonmatching red color cues was present from the fastest responses onward. This is also in line with a contingent-capture account, since control settings are assumed to be set up in advance and can therefore effectively determine which stimuli capture attention directly, from stimulus onset onward (Ansorge \& Horstmann, 2007; Burnham, 2013). In contrast, positive evidence for more deallocation (Theeuwes, 2010; Theeuwes et al., 2000) with nonmatching cues than with matching cues would have consisted of a significant cueing effect by nonmatching cues in the earliest quintile(s) and its decrement with increasing RTs (as more time would have passed since the initial capture). The fact that the predicted interaction between cue type and quintile was not found means that no positive evidence supported the deallocation explanation.

\section{Experiment 2}

In Experiment 2, we tested whether cueing effects of onset cues during search for onset targets depended on a match between cue color and attentional control settings. In Experiment 2a, participants searched for white onset targets and onset cues were of either white or red color. Under the perspective of contingent capture by onsets, both cues should induce a cueing effect, but under the perspective of contingent capture by colors only white onset but not red onset cues should induce a cueing effect.

Above we discussed that it is also possible that participants actively searched for luminance transients. Another possibility is that they searched for colors and onsets simultaneously. Under both these perspectives, in Experiment 1a, the cueing effect for the red onset cue might only be reduced as compared to that of the white onset cue. If participants actively searched for the white onset targets' luminance transients, the red onset cues of a moderate luminance would capture attention to some degree but not as effectively as the white onset cues. On the other hand, if search was directed to both colors and onsets, the white onset cue would capture attention according to a fit to two control settings and, thus, more effectively than the red onset cue that would capture attention only on the basis of its matching to one control setting for onsets.

To discriminate between these two possibilities, in Experiment $2 b$, participants searched for red onset targets, preceded by red onset or white onset cues. The red onset cues should lead to a cueing effect if participants adopted a control setting for color or maybe for onsets and color. The white onset cues should lead to a cueing effect if participants adopted a search setting for onsets or luminance transients only. If participants searched for luminance transients to find the onset targets in Experiments 1a and 1b, we would expect the same results in these experiments: more efficient capture by the white onset cues with their higher luminance than by the red onset cues.

\section{Method}

Participants Sixteen participants were tested in Experiments 2a (12 female, four male; $M_{\text {Age }}=23.25$ years, $S D_{\text {Age }}=2.86$ ) and $2 \mathrm{~b}$ (seven female, nine male; $M_{\mathrm{Age}}=22.20$ years, $S D_{\mathrm{Age}}=$ 1.82), respectively. In Experiment 2b, one participant was excluded due to more than $20 \%$ error trials.

Stimuli and procedure In Experiment 2a, the participants searched for white onset targets, and the cues were either white onset cues or red onset cues. In Experiment 2b, the participants searched for red onset targets, and the cues were either white onset cues or red onset cues. The procedure closely resembled Experiment 1; see also Fig. 3.

\section{Results}

RTs of Experiment 2a Data handling was the same as in Experiment 1, and $5.23 \%$ of all data were removed from the analysis. The cueing effect was systematically influenced by cue type, $F(1,15)=8.23, p=.011$. With a white onset cue, the cueing effect was significantly larger than with a red onset cue (28 vs. $22 \mathrm{~ms})$. We also found a main effect of quintile, $F(4$, $60)=10.92, p<.001$, indicating that the cueing effect increased with the RTs $(17,19,23,28$, and $38 \mathrm{~ms}$, respectively, from the 1 st to the 5 th quintile). Pairwise comparisons showed that the cueing effect of the 5th quintile was significantly larger than in the 1st to 3rd quintiles (all $p$ s $<.002$ ), and the cueing effect in the 4th quintile was significantly larger than that in the 1 st quintile $(p=.040)$. No other effects were found, all $F \mathrm{~s}<1.81$, all $p \mathrm{~s}>.139$; see also Fig. 4.

ERs of Experiment 2a This analysis was analogous to that in Experiment 1. No significant results were found, all $F_{\mathrm{S}}<2.44$, all $p \mathrm{~s}>.139$.

RTs of Experiment 2b Taken together, $6.57 \%$ of all trials were removed. Now the red onset cue $(30 \mathrm{~ms})$ elicited a significantly larger cueing effect than the white onset cue (16 ms), $F(1,14)=19.72, p<.001$. Furthermore, the cueing effect increased with RTs $(16,17,21,26$, and $36 \mathrm{~ms}$, respectively, from the 1 st to the 5 th quintile), $F(4,56)=10.06$, $p<.001$, and was significantly larger in the 5 th quintile than in the 1st, 2nd, and 3rd quintiles (all $p \mathrm{~s}<.015$ ). No other effects were found, all $F_{\mathrm{s}}<0.21$, all $p \mathrm{~s}>.933$, see also Fig. 4 . 

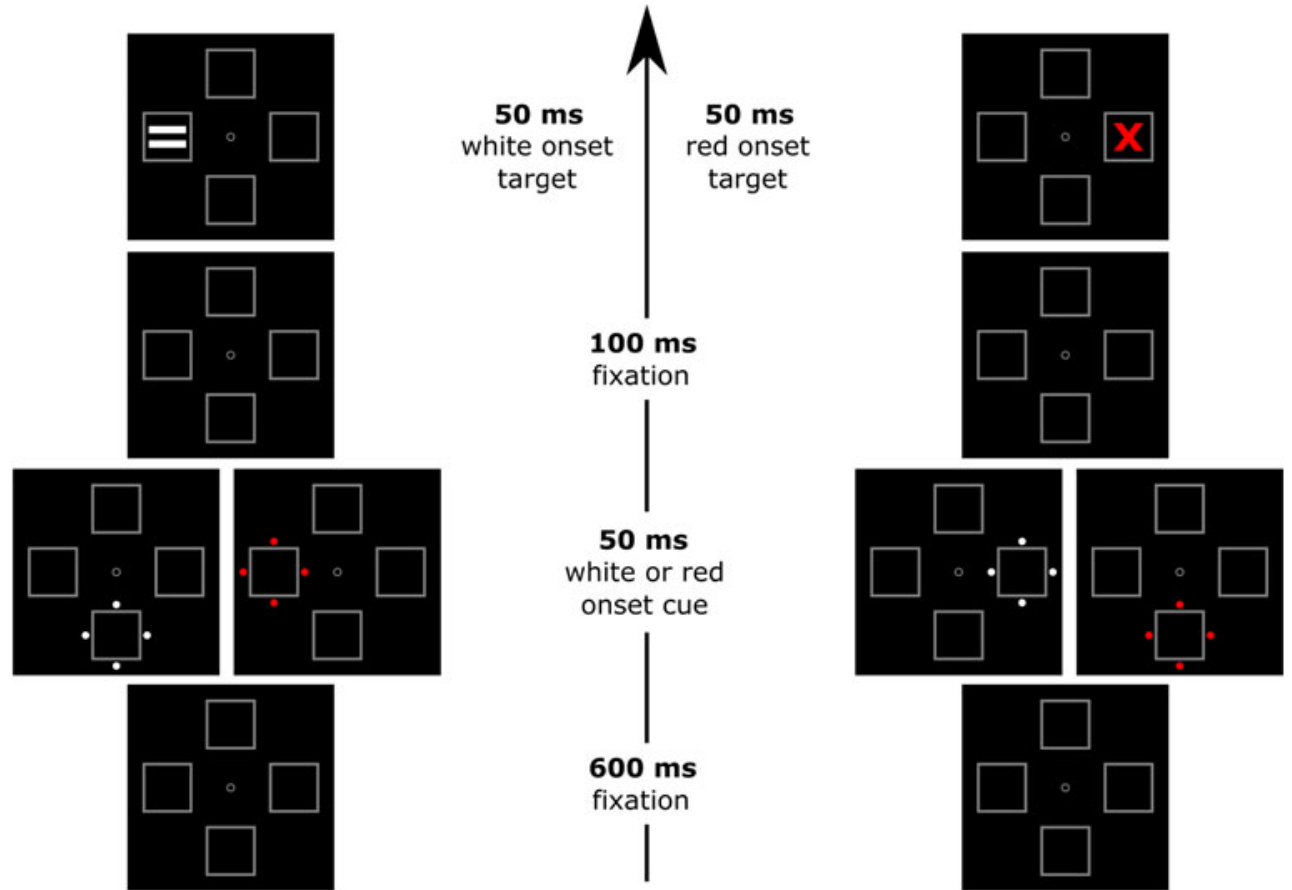

Fig. 3 Example sequences of Experiments 2a (left panel) and 2b (right panel). In Experiment 2a, participants had to identify a white onset target, and in Experiment 2b, they had to identify a red onset target. In both

experiments, the target was preceded equiprobably by a red or a white onset cue. The figure is not drawn to scale

ERs of Experiment 2b ERs were higher with a red onset cue $(7.26 \%)$ than with a white onset cue $(5.17 \%), F(1,14)=$ $11.84, p=.004$. No other effects were found, all $F_{\mathrm{S}}<3.14$, all $p \mathrm{~s}>.584$.

\section{Discussion}

An onset cue of a color similar to the searched-for onset target captured attention more efficiently than a cue of a color dissimilar to the onset target. This was found during search for white onset targets (Exp. 2a) and red onset targets (Exp. 2b). Given that there was no interaction between the variables cue type and quintile, these experiments again provide no direct support for more deallocation of attention following the less similar cues. Furthermore, these results point to a role of topdown attentional control settings for color during search for onset targets and cannot be explained by a control setting for luminance transients. A control setting for luminance transients would have led to more capture by white onset cues than red onset cues, regardless of whether the white onset cues matched (Exp. 2a) or did not match (Exp. 2b) a control setting for colors.

In contrast to Experiment 1b's target-dissimilar color cue condition, here we found significant residual cueing effects of the target-color-dissimilar onset cues (22 and $16 \mathrm{~ms}$ ). Taken in isolation, the residual cueing effect of the color-dissimilar onset cue could be due to a control settings directed to onsets (Folk et al., 1992) or to stimulus-driven capture by just any

abrupt onset (Schreij, Los, Theeuwes, Enns, \& Olivers, 2014; Schreij, Theeuwes, \& Olivers, 2010). When Folk et al. (1992) used a red color target (under otherwise the same conditions as in the present experiment), a white onset cue created a zero cueing effect. This suggests that in the present experiment an attentional control setting for onsets was likely necessary for the residual cueing effects of onset cues with a color different from the target, meaning that participants searched for colors and onsets to find the targets. This interpretation would be in line with other contingent-capture effects, indicative of top-down search for two different features at the same time (Adamo, Pun, Pratt, \& Ferber, 2008; Irons, Folk, \& Remington, 2012). However, this interpretation begs the question as to what might have encouraged the participants to use a top-down set for onsets and for colors in the present experiment because, in contrast to prior studies, it was not necessary to search for both features to find the targets. One reason could be that participants used both of these features in the control settings because both features allowed searching for all targets with equal efficiency. This would imply that sets for color should not play a role during search for onset targets, in which color search would be less efficient (see Exp. 5).

\section{Experiment 3}

In Experiments 1 and 2, the red cues and targets had lower luminance transients and contrast than the white cues and 

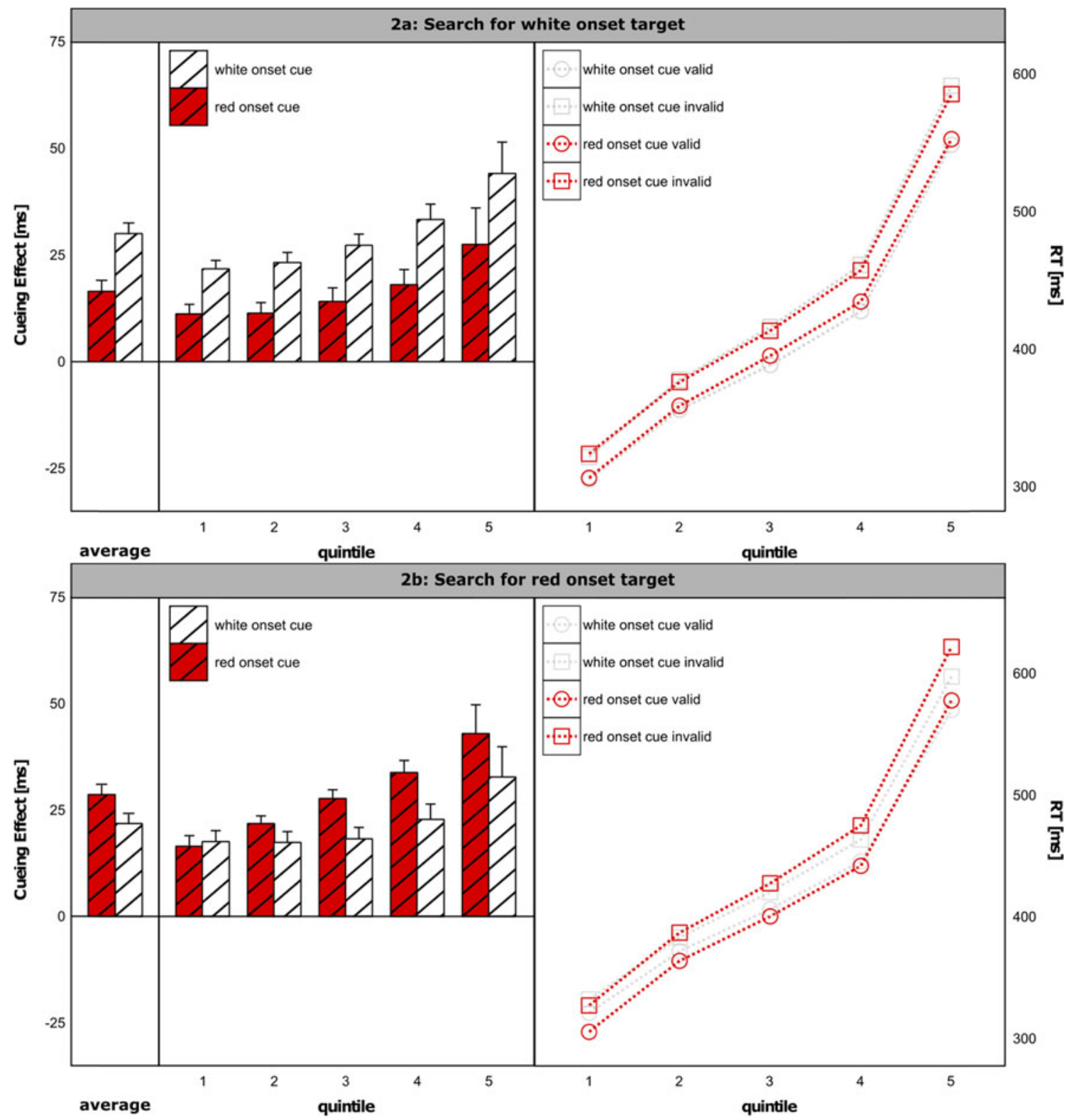

Fig. 4 The top panel depicts the results of Experiment 2a, and the bottom panel those of Experiment $2 b$

targets. Therefore, we tested whether the results of Experiment 2 would hold with the contrast and strength of the luminance transient equated for achromatic onset cues and onset target colors (see Fuchs et al., 2013). Participants searched for light onset targets (Exp. 3a) or for dark onset targets (Exp. 3b), and the cues in both experiments were light or dark. If top-down control settings are directed to target onsets or luminance transients, we expected both kinds of onset cues to capture attention to similar extents in Experiments $3 \mathrm{a}$ and $3 \mathrm{~b}$ because the strengths of the luminance transients of the light and the dark stimuli were carefully equated for their resulting contrasts. The same prediction would be made on the basis of stimulus-driven capture by all onset cues and on the basis of capture contingent on control settings for onset singletons.
However, if control settings were directed to achromatic target colors (much as to target chromaticity in Exp. 2b), we would expect cueing effects by light but not by dark onset cues during search for light onset targets, and by dark but not by light onset cues during search for dark onset targets.

\section{Method}

Participants Sixteen participants were tested in Experiment 3a (ten female, six male; $M_{\mathrm{Age}}=21.69$ years, $S D_{\mathrm{Age}}=3.44$ ), and another 16 participants (nine female, seven male; $M_{\text {Age }}=$ 22.36 years, $S D_{\text {Age }}=6.03$ ) were tested in Experiment $3 \mathrm{~b}$. In Experiment $3 b$, two participants were excluded owing to more than $20 \%$ errors. 


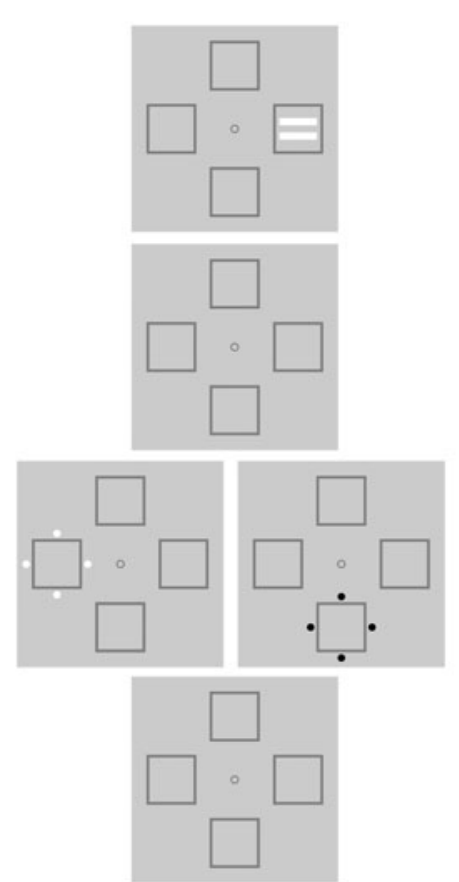

Fig. 5 Example sequences of displays in trials of Experiments 3a (left panel) and 3b (right panel). In Experiment 3a, participants had to identify a light onset target, and in Experiment $3 b$ they

Stimuli and procedure The stimuli and procedure were the same as in Experiment 2, with the following exceptions; see also Fig. 5. The background was gray $\left(75.5 \mathrm{~cd} / \mathrm{m}^{2} ;-5.3 /-18.8\right)$. The dark onset cues of Experiments $3 \mathrm{a}$ and $3 \mathrm{~b}$, as well as the placeholders and the central fixation point in Experiment $3 \mathrm{~b}$, had a luminance of $13.3 \mathrm{~cd} / \mathrm{m}^{2} ;-1.8 /-9.1$. The light onset cues of Experiments $3 \mathrm{a}$ and $3 \mathrm{~b}$, as well as the placeholders and the central fixation point in Experiment $3 \mathrm{a}$, had a luminance of $137.7 \mathrm{~cd} / \mathrm{m}^{2} ;-7.8 /-28.4$. The dark and light stimuli were thus of equal stimulus-background Weber contrasts ( \pm 0.82$)$ and created equally strong luminance transients.

\section{Results}

RTs of Experiment 3a Overall, $6.46 \%$ of all trials were removed. The significant main effects of quintile, $F(4,60)=$ $6.37, p=.016$, and cue type, $F(1,15)=12.84, p=.002$, are both best explained in terms of their interaction, $F(4,60)=$ $4.34, p=.004$. For target-color similar light onset cues, the cueing effect grew with RTs $(21,26,32,38$, and $61 \mathrm{~ms}$, respectively, for the 1 st to the 5 th quintile) and was significantly different in the 5 th quintile than in the 1 st, 2nd, and 3rd quintiles (all $p$ s $<.028$ ). For the target-color dissimilar dark onset cues, the cueing effect differed nonsignificantly between 14 and $18 \mathrm{~ms}$. In each quintile, the cueing effect of the light onset cue was significantly larger than the cueing effect of the dark onset cue (all $p \mathrm{~s}<.022$ ). In addition, the cueing effect for the dark onset cue was not significantly different from zero in the fifth quintile, $t(15)=-1.09, p=.292$. For these results, see also Fig. 6.

ERs of Experiment 3a Participants made more errors in invalid $(6.26 \%)$ than in valid $(5.17 \%)$ trials, $F(1,15)=$ 9.96, $p=.007$. No other effects were found, all $F_{\mathrm{S}}<4.33$, all $p \mathrm{~s}>.521$.

RTs of Experiment 3b After the exclusion of errors (6.11 \%), the ANOVA showed significant main effects of quintile, $F(4,52)=3.17, p=.028$, and cue type, $F(1,13)=$ $21.99, p<.001$, but no interaction $(p=.149)$. The cueing effect was significantly larger for target-colorsimilar dark $(32 \mathrm{~ms})$ than for dissimilar light onset cues (13 ms). Furthermore, the cueing effect grew numerically with RTs $(16,18,21,27$, and $32 \mathrm{~ms}$, respectively, from the 1 st to the 5 th quintile), but none of these differences were significant (all $p \mathrm{~s}>.110$ ).

ERs of Experiment $\mathbf{3 b}$ The ERs were higher in invalid $(5.70 \%)$ than in valid $(4.65 \%)$ trials, $F(1,13)=5.47$, $p=.036$. Furthermore, we observed a main effect of cue type, $F(1,13)=7.39, p=.018$. Participants made more errors with dark $(5.69 \%)$ than with light $(4.66 \%)$ onset cues. 

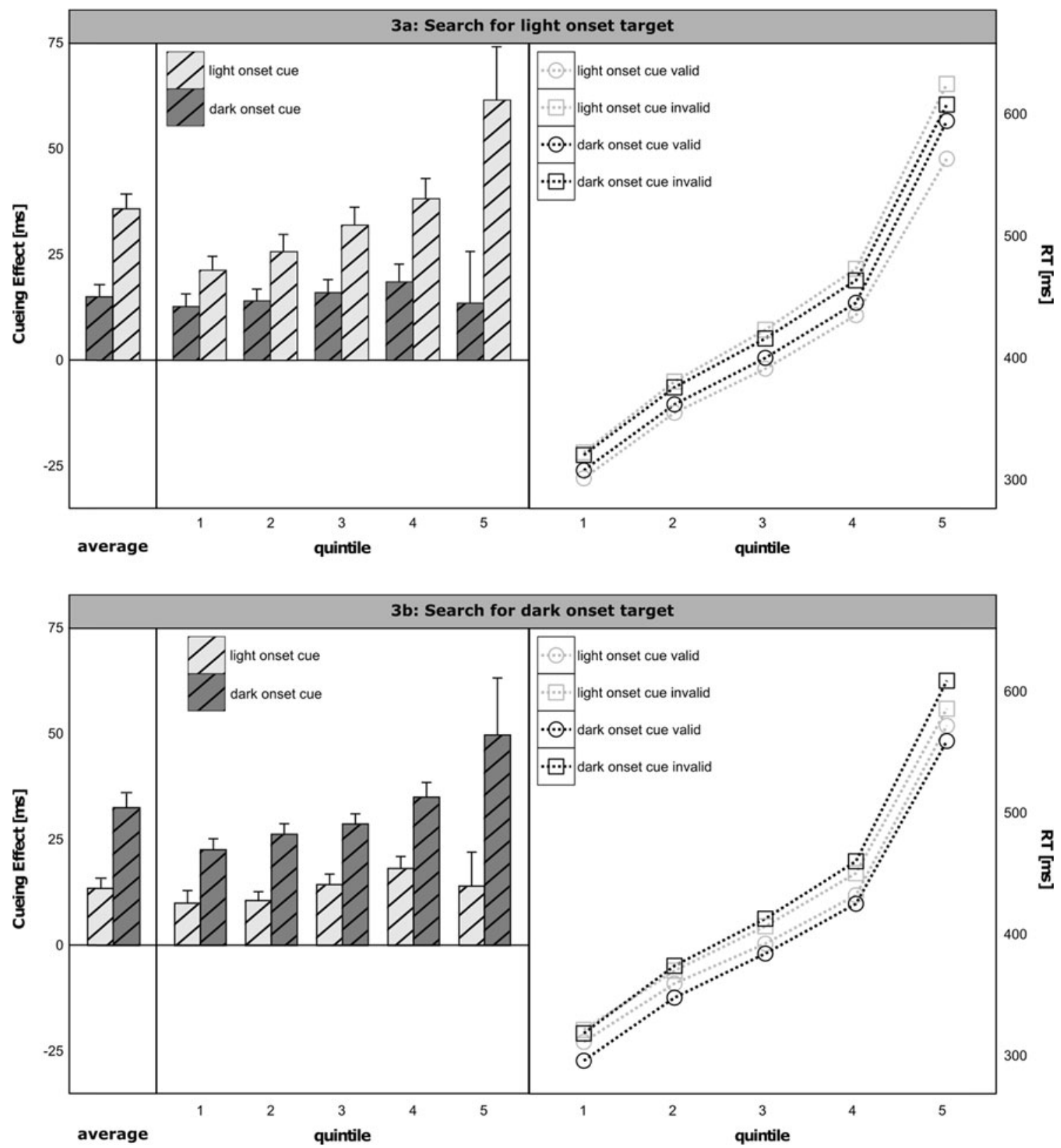

Fig. 6 The top panel depicts the results of Experiment 3a, and the bottom panel those of Experiment $3 b$

\section{Discussion}

Experiment 3 showed that when participants searched for an onset target of a particular achromatic color, onset cues of this particular color led to a stronger cueing effect than did onset cues of a different color but of equally strong contrast and luminance transient. During search for a light onset target, light onset cues captured attention more efficiently than dark onset cues (Exp. 3a), but during search for a dark onset cue this pattern reversed, and dark onset cues captured attention more efficiently than light ones (Exp. 3b). This pattern conceptually replicates the findings of Experiment 2 and again shows that the use of a color-directed attentional control setting does not critically depend on the selective presence of a stronger luminance transient (or contrast difference) at the position of the onset cues. In addition, as in Experiment 2 , we found residual cueing effects by onset cues of a color dissimilar to the searched-for target.

These results are only partly in line with prior findings by Fuchs et al. (2013), in which even reversed cueing effects for the less-matching target-color-dissimilar dark and light onset cues were found. A number of methodological differences might be responsible for the different results. For example, Fuchs et al. (2013) used only two possible cue and target positions, whereas in the present study four target positions were possible. Active suppression of an irrelevant color cue 
might be easier with just two than with four potential cue positions. Also, the cue-target interval was shorter $(16 \mathrm{~ms})$ in Fuchs et al.'s than in the present study $(150 \mathrm{~ms})$. It is possible that a closer temporal proximity of the cue and target calls for more active suppression of the irrelevant cues, or that it took a while for the residual capture effect of the lessmatching cues to build up, so that this was overlooked with the short interval. However, this conclusion is not supported by the present results, because the cueing effect of the lessmatching dark cue in Experiment $3 \mathrm{a}$ did not increase as a function of RTs.

In contrast to Experiments 1 and 2, we also found an interaction between cue type and quintile, at least in Experiment $3 \mathrm{a}$, but this interaction was not predicted by the deallocation account. The interaction was due to an increasing cueing effect in color-matching cue conditions across the quintiles of the RT distribution. Again, in line with a contingent-capture explanation, already among the fastest responses, the cueing effect of the light onset cue that would have matched a topdown control setting for target colors was stronger than that of the dark onset cue, which would not have matched the same control setting.

\section{Experiment 4}

Experiment 4 was a conceptual replication of Experiment $1 \mathrm{~b}$, but with luminance-equated light and dark color cues. In Experiment $4 \mathrm{a}$, a light onset target was used, and in Experiment $4 \mathrm{~b}$, a dark onset target was used. In both experiments the color cues were equally likely to be light or dark. If contingent capture by color was at work, a cueing effect of light color cues (in Exp. 4a; of dark color cues in Exp. 4b), but not of dark color cues (in Exp. 4a; of light color cues in Exp. $4 b)$ would be expected. In contrast to these predictions, on the basis of a top-down control setting for onsets, we would expect to find no cueing effects with any of the color cues. Finally, if the participants used an attentional control setting for singletons, or if just any singleton captured attention in a stimulus-driven way, a cueing effect in all of the cue type conditions would be expected.

\section{Method}

Participants Sixteen participants were tested in Experiments 4a (nine female, seven male; $M_{\mathrm{Age}}=23.69$ years, $S D_{\mathrm{Age}}=$ 3.59 ) and $4 \mathrm{~b}$ (ten female, six male; $M_{\text {Age }}=23.73$ years, $S D_{\text {Age }}=9.11$, respectively.

Stimuli and procedure The stimuli and procedure were the same as in Experiment 3, with the exception of additional nonsingletons that were used in the displays (see Fig. 7): With a dark color cue, white nonsingletons were presented around all three of the remaining placeholders, and with a light color cue, dark nonsingletons were shown around all three of the remaining placeholders.

\section{Results}

RTs of Experiment 4a Due to the usual data handling, $6.50 \%$ of all trials were excluded. A main effect of cue type, $F(1,15)=142.28, p<.001$, was further modulated by its significant interaction with quintile, $F(4,60)=3.82, p=.008$. For the light color-matching cue, the cueing effect increased with RTs $(32,33,34,36$, and $42 \mathrm{~ms}$, respectively, from the $1 \mathrm{st}$ to the 5 th quintile), although none of the differences between quintiles were statistically significant (all $p \mathrm{~s}>.790$ ). For the dark color-nonmatching cue, on the other hand, the cueing effect became numerically more negative $(-13,-15,-15$, -17 , and $-25 \mathrm{~ms}$, respectively, from the $1 \mathrm{st}$ to the 5 th quintile), but the differences were again not significant, all $p \mathrm{~s}>.200$. In each quintile, the cueing effects of the light colormatching cues were significantly larger than that of the dark color nonmatching cues, all $p \mathrm{~s}<.001$. For the results, see also Fig. 8.

ERs of Experiment 4a We observed no significant effects in the ERs, all $F \mathrm{~s}<2.25$, all $p \mathrm{~s}>.154$.

RTs of Experiment $4 \mathrm{~b}$ Out of all trials, $5.82 \%$ were removed from the analysis. We obtained results analogous to those of Experiment 4a: a main effect of cue type, $F(1,14)=$ 106.83, $p<.001$, that interacted with quintile, $F(4,56)=$ $3.99, p=.006$. In each quintile, the cueing effect of the matching dark color cue was stronger than that of the nonmatching light color cue, all $p$ s $<.001$. However, the interaction was due to the fact that the cueing effect of the dark color cues grew across RTs $(24,26,30,31$, and $39 \mathrm{~ms}$, respectively, from the 1 st to the 5 th quintile), with a significant difference between the 1 st and 5th quintiles, $p=.019$. In contrast, for the light color cues, the cueing effect was more constant across RTs $(-8,-7,-8,-9$, and $-9 \mathrm{~ms}$, respectively, from the 1 st to the 5 th quintile), but it was not significantly different from zero in the fifth quintile, $t(14)=-1.71, p=.110$.

ERs of Experiment $4 \mathbf{b}$ The analysis of the ERs yielded no significant results, all $F_{\mathrm{S}}<2.00$, all $p \mathrm{~s}>.179$.

\section{Discussion}

Experiment 4 confirmed the findings of Experiment $1 \mathrm{~b}$ : There was a cueing effect for light cues when participants searched for light targets, and for dark cues when participants searched for dark targets. In other words, the cueing effect was restricted to the top-down matching color cues. However, we even found reversed cueing effects, indicative of an active 


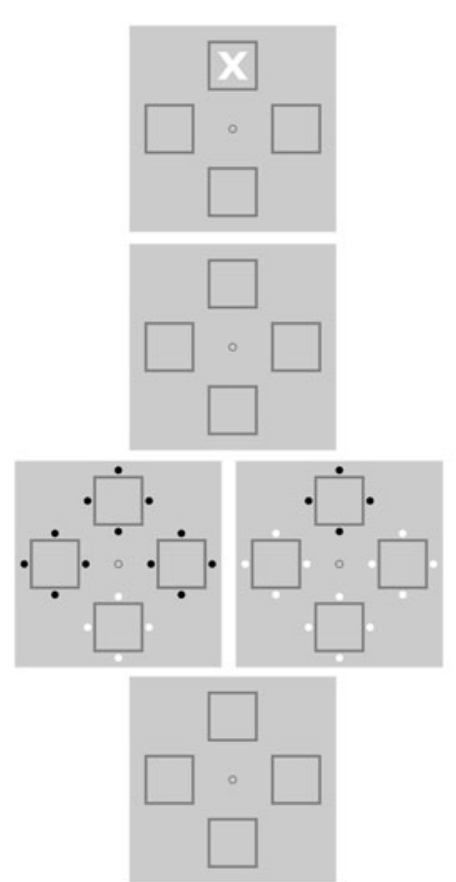

Fig. 7 Example sequences of displays in trials of Experiments $4 \mathrm{a}$ (left panel) and $4 \mathrm{~b}$ (right panel). In Experiment 4a, participants had to identify a light onset target, and in Experiment $4 \mathrm{~b}$ they

suppression of the cues, if a nonmatching color cue was used. Together, these results cannot be explained by a set for luminance transients, because the strengths of the luminance changes were equated for the resulting contrasts in all conditions.

Additionally, the presence of a stronger cueing effect for top-down matching color cues than for nonmatching color cues throughout the RT distribution (from the fastest responses onward) is in line with a contingent-capture explanation. Again, however, as was noted already with respect to Experiment $1 \mathrm{~b}$, the presence of the nonmatching color cues might have invited the participants to select an attentional control setting for target colors instead of an attentional control setting for onsets. With an attentional control setting for colors, participants would have achieved two goals at the same time: They would have directed their attention to $100 \%$ of the targets, and they would have ignored $50 \%$ of the uninformative color cues - namely, the nonmatching cues with a color different than that of the targets.

\section{Experiment 5}

Taken together, Experiments 1-4 painted a relatively homogeneous picture: Cue-target color similarities contribute to attention capture by onset cues. However, in Experiments 2 and 3, it remained unclear what mechanism accounted for the residual cueing effects of the onset cues with a color different

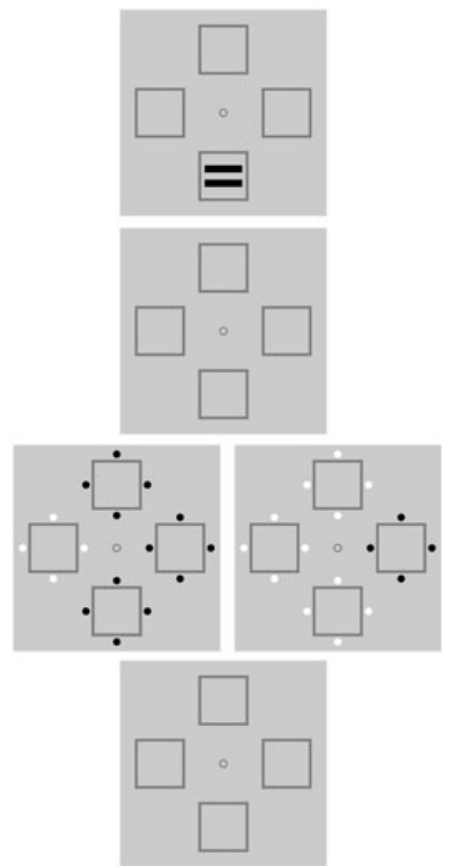

had to identify a dark onset target. In both experiments, the target was equiprobably preceded by a light or a dark color cue. The figure is not drawn to scale

from that of the onset targets. Following Experiment 2, we speculated that participants could have equally efficiently searched for the targets by their onsets or by a single target color. In general agreement with this possibility, recent findings have shown that participants base their inclusion of more than one feature into attentional control settings on the context-for instance, on the efficiency with which a feature can be used to find the targets (Mast \& Frings, 2014).

To see whether context and efficiency affected the use of color or onsets in attentional control settings, and to get an idea of the origin of the residual cueing effects by onset cues with a target-dissimilar color in the present Experiments 2 and 3, we used two tasks in different blocks. In the "onset-target block" of Experiment 5, participants searched for two differently colored (red and white) onset targets simultaneously. In each trial, one of the two targets appeared randomly. Under these conditions, it was more efficient for the participants to search for onsets only than for colors, because it was possible to find all targets by searching for the single feature of onset. Hence, a top-down set for onsets was encouraged, and a topdown set for colors discouraged. If, under these conditions, participants searched for onsets only, we would expect to see similar cueing effects for both onset cues: cues with a targetsimilar color (e.g., red cues) and cues with a color dissimilar to all targets (blue cues, since the targets were red or white).

If, however, colors are an inherent property of any topdown set for onsets, participants should have continued to search for the particular target colors under the present 

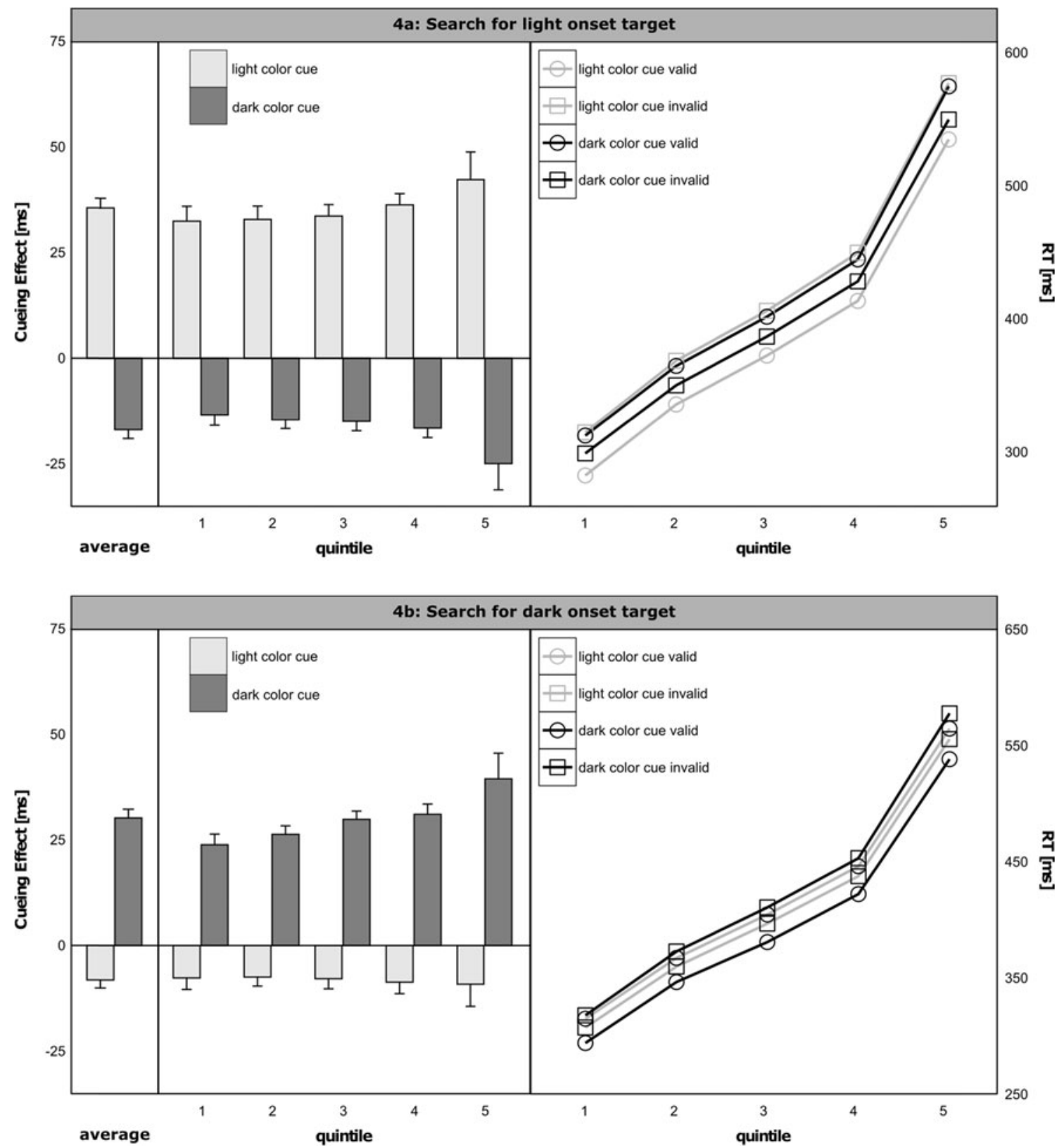

Fig. 8 The top panel depicts the results of Experiment 4a, and the bottom panel those of Experiment $4 \mathrm{~b}$

conditions, and we would have expected to observe a stronger cueing effect for onset cues of a target-similar color than for onset cues of a target-dissimilar color. In fact, if participants only searched for the two target colors and not at all for onsets, we would expect to find zero cueing effects by the cues of a target-dissimilar color. However, this is an unlikely outcome in light of the results of Experiments 2 and 3, in which onset cues of a target-dissimilar color captured attention at least to some degree.

To further explore the hypothesis that efficiency determined the inclusion of color in the top-down set, and to better understand the origin of the residual cueing effect by onset cues of a target-dissimilar color, we used a second blocked task (the "white-target block") with onset and color targets, in which a top-down set for color was encouraged. In this whitetarget block, participants searched for white onset targets and white color targets at the same time (because these two target types were presented in an unpredictable manner). If the efficiency of top-down search for onsets was responsible for the residual cueing effects of onset cues with a target-dissimilar color in Experiments 2 and 3, in the present situation, participants should have preferentially searched for the single color to find all of the targets, rather than keeping top-down sets for both onsets and colors. This prediction was tested by the use of two different onset cues in the second blocked condition: white onset cues with a target-similar color, and red onset cues 
with a target-dissimilar color. If, under these conditions, participants abandoned their top-down sets for onsets entirely, we would find a cueing effect by onset cues with a target-similar color (i.e., the white onset cues), but a zero cueing effect by the onset cues with a target-dissimilar color (i.e., the red onset cues). Note how this prediction differs if the residual cueing effect of onset cues of a target-dissimilar color were due to stimulus-driven capture: If any onset cue captures attention to some degree, we would expect to see a (reduced but significant) cueing effect even for the onset cue with a targetdissimilar color.

The second block also allowed us to test yet another contribution to the residual cueing effect of the onset cues with a target-dissimilar color in Experiments 2 and 3: If the presence of an onset target in trial $n-1$ primed capture by an onset cue in the following trial $n$, we should find evidence of residual cueing effects of the onset cues with a target-dissimilar color, at least in those trials $n$ that followed an onset target in $n-1$, but less so, or maybe not at all, in those trials $n$ that followed a color target in $n-1$ (cf. Awh, Belopolsky, \& Theeuwes, 2012).

\section{Method}

Participants, stimuli, and procedure Because the residual cueing effect of the onset cues with a target-dissimilar color was expected to further decrease (maybe to zero), in comparison to Experiments 2 and 3, the number of participants was increased to also increase the power of our test for the residual cueing effects. Twenty-six participants were tested (15 female, 11 male; $M_{\text {Age }}=22.85$ years, $\left.S D_{\text {Age }}=4.15\right)$. The stimuli were the same as in Experiments 1 and 2, except as noted. The experiment consisted of two different blocks (see also Fig. 9). In the onset-target block, each trial could feature either a red or a white onset target. Both targets were equally likely and randomly intermixed. The targets were preceded by either a red or a blue $\left(25.8 \mathrm{~cd} / \mathrm{m}^{2}\right.$; CIE Lab: 49.7/-98.5) onset cue. In the white-target block, we used white onset targets (that were presented in isolation) and white color targets (that were presented simultaneously with red distractors in the remaining placeholders). Targets were equally likely to be preceded by red or white onset cues.

\section{Results}

RTs Data handling was the same as in Experiment 1 and led to the exclusion of $6.91 \%$ of all trials. We conducted separate analyses for the two experimental blocks. Each ANOVA contained the within-subjects variables RT quintile (1-5) and cue type (red vs. blue onset cue in Block 1; white vs. red onset cue in Block 2).

In the onset-target block, we only found a significant effect of RT quintile, $F(4,100)=17.17, p<.001$. The cueing effect increased across quintiles $(17,20,24,30$, and $53 \mathrm{~ms})$, but the only significant differences were found between the fifth and all other quintile (all $p \mathrm{~s}<.001$ ). No other significant results were found, all $F \mathrm{~s}<.21$, all $p \mathrm{~s}>.65$.

The white-target block yielded a different result. We found a main effect of cue type, $F(1,25)=130.66, p<.001$, indicating that the cueing effect elicited by white onset cues (30 ms) was larger than the cueing effect elicited by red onset cues $(-5 \mathrm{~ms})$. The cueing effect of the red onset cues was not different from zero, $t(25)=-1.80, p=.084$. Additionally, we found a main effect of quintile, $F(4,100)=17.44, p<.001$, indicating an increase of the cueing effect across RTs $(3,7,12$, 16 , and $24 \mathrm{~ms}$, respectively, from the $1 \mathrm{st}$ to the 5 th quintile). The cueing effect in the first quintile was not different from zero, $t(25)=1.39, p=.180$. The cueing effect in the fifth quintile was significantly larger than those in the first quintile $(p<.001)$ and the second quintile $(p=.011)$. Figure 10 illustrates the results.

Despite the facts that the color-dissimilar cue in the whitetarget block did not lead to significant cueing effects and that the conditions for a test of the origin of the residual cueing effect of these cues were not ideal, we conducted a repeated measurements ANOVA, with the cueing effect as a dependent variable and cue type, target type, and target type in trial $n-1$ as independent within-subjects variables. Besides the results reported above, we found an interaction between cue type and target type in trial $n-1, F(1,25)=4.96, p=.035$. In trials with red onset cues, there was a small cueing effect following a white onset target in $n-1$ ( $7 \mathrm{~ms})$, but an inverted cueing effect after a white color target in $n-1(-8 \mathrm{~ms}), t(25)=2.31, p=.029$. This result supports the hypothesis that the residual cueing effect of red onset cues is due to intertrial priming of attention. Also of interest, in trials with white onset cues, the cueing effects were similar regardless of whether a white onset target (30 ms) or a white color target $(34 \mathrm{~ms}), t(25)=-0.72, p=.480$, had been shown in trial $n-1$. This finding points to independent origins of top-down contingent capture by color (here, of the white cues) and of capture by cues with a feature that was primed by a target in $n-1$.

ERs The arcsine-transformed ERs were subjected to a repeated measurements ANOVA, with the within-subjects variables validity, cue type, and experimental block. We only found a main effect of validity, $F(1,25)=18.97, p<.001$, with higher ERs with invalid $(6.58 \%)$ than with valid $(5.61 \%)$ cues. Additionally, a strong trend toward an effect of experimental block was apparent, $F(1,25)=4.20, p=.051$, with higher ERs in the white-target block $(6.88 \%)$ than in the onset-target block $(5.30 \%)$. No other effects were found, all $F_{\mathrm{S}}<3.49$, all $p \mathrm{~s}>.073$.

\section{Discussion}

In the onset-target block, during search for red and white onset targets, blue target-color-dissimilar cues and red target-colorsimilar onset cues captured attention with similar efficiency. 


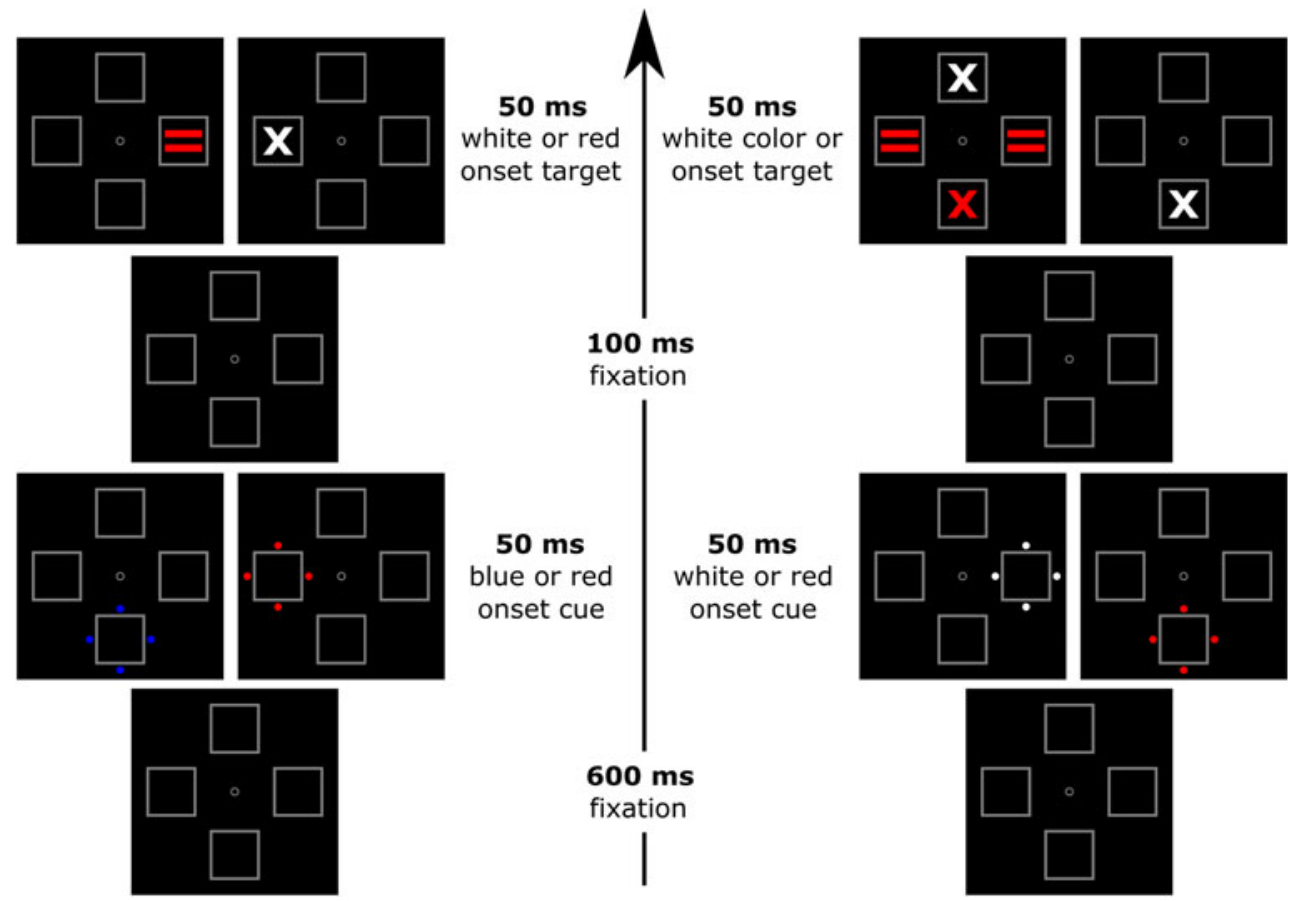

Fig. 9 Example sequences of displays in trials of Experiments 5. The sequence on the left depicts the onset-target block, in which participants searched for a red and a white onset target. These target displays were preceded by red or blue onset cues. The panel on the right depicts the

Cue-target color similarity therefore did not result in a stronger cueing effect. This indicates that participants probably adopted an attentional control setting for onsets rather than for different colors. The results are in line with a role of efficiency considerations among the participants when they were setting up their attentional control settings. If all targets could be efficiently found by a searching for onsets but if different target colors would imply searching for different colors, participants seemed to use onsets rather than colors to search for the targets. In the present experiment's onset-target block, search for onsets was more efficient than search for colors, so the fact that cue-target color similarities did not play a role for the cueing effect supports a top-down explanation of color contributions to the cueing effect of onset cues.

In contrast to the onset-target block, the white-target block of the present experiment showed that participants adopted an attentional control setting for color. This attentional control setting for color was apparently very effective in eliminating the residual cueing effect of the onset cues with a targetdissimilar color: On average, in the white-target block we found no cueing effect of the red onset cues, although half of the trials did contain a (white) onset target. We think that the absence of an average cueing effect of the red onset cues, which contrasts with the residual cueing effect of the same cues in Experiment 2a, is due to the fact that an attentional control setting for one color was necessary and sufficient to search for all targets of Experiment 5. white-target block, in which participants searched for a white onset target and a white color target. Targets were preceded equiprobably by red or white onset cues. The figure is not drawn to scale

On closer inspection, however, we found that a small cueing effect of the red onset cues might have been obtained in target-color blocks in which an onset target in the preceding trial primed attention capture by an onset cue in the immediately following trial. This finding is in line with the observation that intertrial priming of attention by feature repetitions (Maljkovic \& Nakayama, 1994) is one source of cueing effects (Belopolsky, Schreij, \& Theeuwes, 2010). Additionally, the same intertrial priming effects were not found with the white onset cues. It seems that intertrial priming of attention could not account for the cueing effect of the onset cues with a target-similar color. Instead, these cues probably captured attention in a top-down way - that is, due to their color's match to the top-down control settings set up to search for the white targets, and regardless of whether these features were primed by target features in $n-1$.

\section{General discussion}

In the present research, we set out to study a simple question: Are cueing effects in onset-target conditions of contingentcapture experiments due to attentional control settings directed to abrupt onsets, or are they due to control settings directed to target colors? The results clearly supported a role of attentional control settings for colors. When participants were searching for a white onset target, we found a cueing effect 

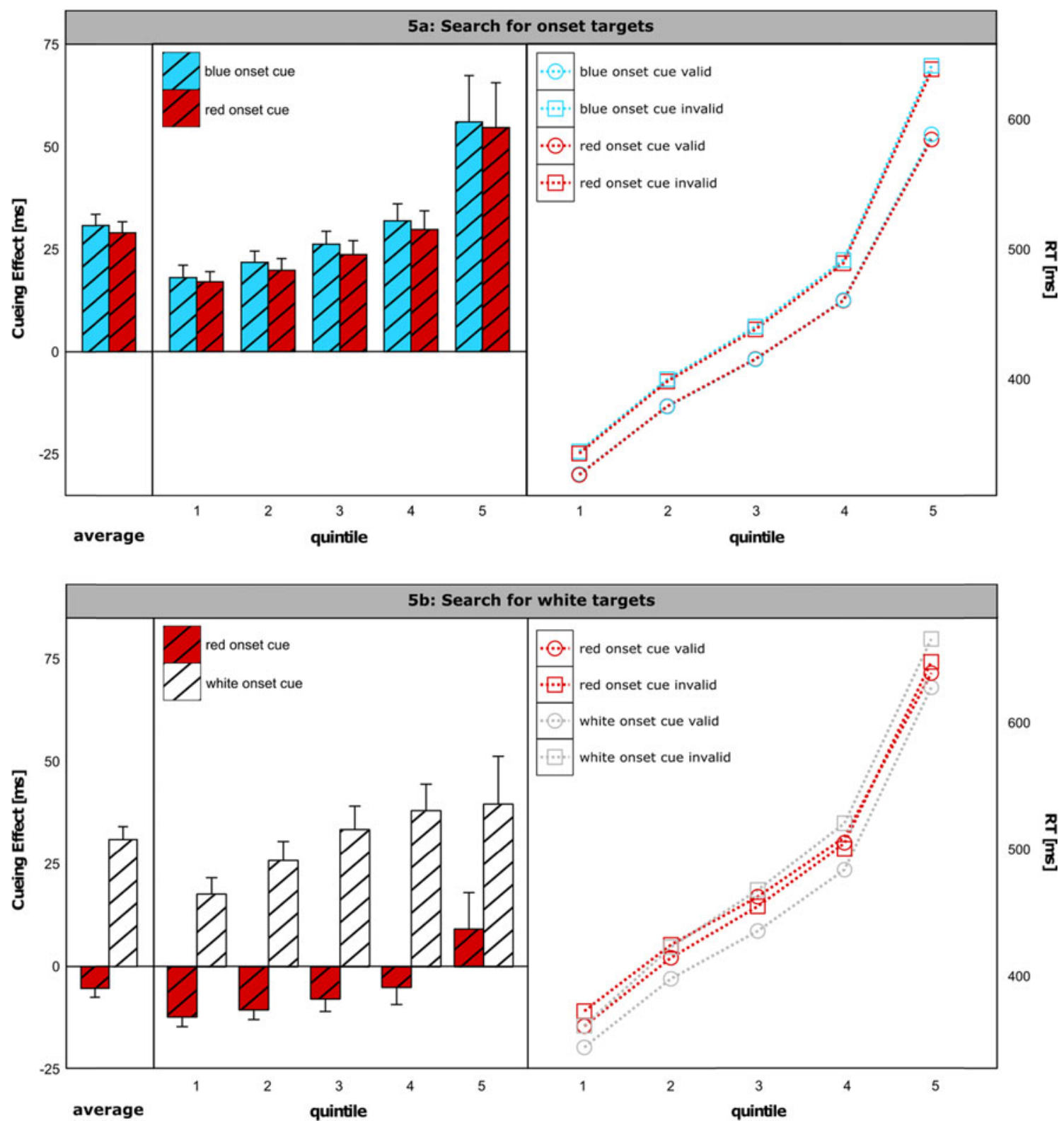

Fig. 10 The panel at the top depicts performance in the onset-target block of Experiment 5, and the bottom panel depicts performance in the white-target block of Experiment 5

of white onset cues as well as of white color cues, although the white color cues were presented together with red color distractors (in Exps. 1a and 1b) or with dark color distractors (in Exp. 4a), so that it was impossible for these color cues to capture attention by their onsets alone. In contrast to the white color cues, red color cues (in Exp. 1b) or black color cues (in Exp. 4a) did not induce cueing effects during search for white onset targets. Thereby, explanations in terms of a stimulusdriven capture effect by just any pop-out stimulus (e.g., Theeuwes, 2010), or in terms of a contingent-capture effect based on an attentional control setting for such pop-out singletons (e.g., Bacon \& Egeth, 1994), were ruled out as potential alternative explanations. Also, in control conditions with dark onset targets, the pattern of results was reversed, so that standard cueing effects were restricted to dark color cues and absent for light color cues (Exp. 4b).

In addition, we also found stronger cueing effects of onset cues that had a color similar to the searched-for onset target. This stronger cueing effect was found relative to onset cues with a color dissimilar to that of the onset target. For example, when the participants searched for a red onset target, we observed stronger cueing effects of red than of white onset cues, a pattern that reversed during search for white onset targets (Exp. 2). Analogous results were found during search for light versus dark onset targets (Exp. 3). These experiments showed that participants might have used top-down control settings 
directed to onset targets (at least on some of the trials), but that top-down control settings directed to target colors were probably also at work.

This interpretation was supported in Experiment 5. In Experiment 5, participants either searched for two onset targets of different colors or for two white targets, half of which were onset targets. Under these conditions, attentional control settings for onsets and for the color white, respectively, were encouraged. In line with the view that residual capture by targetcolor-dissimilar onset cues in Experiments 2 and 3 were due to control settings for onsets, in Experiment 5 color-dissimilar onset cues did not capture attention during search for white onset and color targets, but they did capture as much attention as color-similar onset cues during search for red and white onset targets. Together, these results suggest that even the residual cueing effect of the target-dissimilar cues in Experiments 2 and 3 was contingent on the participants' use of an appropriate top-down attentional control setting - a conclusion that has also been reached on prior research (Folk et al., 1992).

Furthermore, the stronger cueing effects of cues with a color matching that of the searched-for targets than of cues with a color different from the targets were present from the fastest responses onward. This observation is in line with the theory of top-down contingent capture because, according to this theory, participants would set up their attentional control settings in advance, so that each stimulus that matched the setting would have a chance of capturing attention more efficiently from the fastest responses onward (Ansorge \& Horstmann, 2007; Burnham, 2013). In the present study, we found no direct support for the deallocation theory, which predicts an increase of the cueing-effect difference between matching and nonmatching cues across time. Of course, it is possible that the cue-target interval was simply too long for this predicted pattern of deallocation. For example, on the basis of the data of Kim and Cave (1999), one would expect that deallocation could have taken effect within $100 \mathrm{~ms}-\mathrm{a}$ time considerably shorter than the 150 -ms interval between cue and target that was used here. Thus, theoretically, deallocation might have already taken place even before the fastest responses in the present study. Note, however, that other studies that have varied the cue-target interval showed contingent-capture effects-stronger cueing effects of topdown matching than of nonmatching cues-with much shorter intervals (Ansorge \& Heumann, 2003; Ansorge \& Horstmann, 2007; Remington, Folk, \& McLean, 2001). The conclusion that deallocation does not play a prominent role is also supported by event-related potential studies. In such studies, attentional capture by the cue can be continuously tracked with millisecond precision from cue onset onward, and generally, no evidence of early capture by nonmatching color cues can be observed in such studies (Ansorge, Kiss, Worschech, \& Eimer, 2011; Eimer \& Kiss, 2008; see also McDonald, Green, Jannati, \& Di Lollo, 2013).
One might also question whether the stronger cueing effect of the top-down matching color cues reflected (only) contingent capture. It might equally well be that intertrial priming of capture contributed to the cueing effect (Awh et al., 2012). According to this theory, the allocation of attention to a target in a preceding trial $n-1$ might have boosted attention capture by a similarly colored top-down matching cue in the current trial $n$ (cf. Maljkovic \& Nakayama, 1994; Theeuwes, 2013). Note that a corresponding priming of capture could not have taken effect with those nonmatching cues that were always of a color different from that of the preceding target. In Experiments 1-4 of the present study, it was impossible to test for intertrial priming because only one target was used, and therefore the same cue types were primed in each and every trial. On the basis of the existing literature, however, it is clear that the intertrial priming of capture does not account for the full strength of the contingent-capture effect (see Ansorge \& Horstmann, 2007; Folk \& Remington, 2010; Worschech \& Ansorge, 2012; for a review, see Lamy \& Kristjánsson, 2013). Also, as the results of Experiment 5 show, during search for onset targets, the residual cueing effect of onset cues with a nonmatching color can be attributed to intertrial priming, but not that of the onset cues with a matching color.

The results of Experiment 5 also suggest one other consideration. It has been suggested that onsets (transient features) elicit stimulus-driven capture of attention, whereas color elicits topdown contingent capture (Liao \& Yeh, 2013). The results of Experiments 2 and 3 would be in line with this explanation. Onsets could have captured attention automatically, and color would have worked on top of this automatic capture. However, as Experiment 5 showed, the residual capture effect of the onset cues in the present study was a consequence of top-down sets. If a target-color search was suggested by its greater economy (as in Exp. 5's white-target block), no capture by onset cues of a nonmatching color was found, unless an onset target in the preceding trial had primed capture by an onset cue in the immediately following trial. Yet it is possible that more evidence for a stimulus-driven cueing effect of onset cues with a targetdissimilar color not matching the control settings could be found with a shorter cue-target interval than was used here.

In a broader perspective, our results can be regarded as further evidence that top-down attentional control settings are not as simple as has previously been assumed. For example, recently the idea has been advocated that control settings might be directed to combinations of features, such as color and position (Adamo et al., 2008) or different colors (Irons et al., 2012), or even that semantic categories could be used to selectively attend to particular classes of stimuli (Wyble, Folk, \& Potter, 2013). Along similar lines, it has been shown that control settings vary as a function of the task context - that is, color could be included flexibly within control settings, depending on how helpful color is for telling apart targets from distractors (Mast \& Frings, 2014), and even control settings 
for nonmatching cues seem to be flexibly used by participants when recent cue utility suggests doing so (Goller \& Ansorge, 2015). These findings were generally supported by the present research, in which we showed that attentional capture can depend on both onset and color information (Exps. 2 and 3), and that it seems to be a question of how efficiently the targets can be selected that determines which features are incorporated into top-down attentional control settings (Exp. 5).

\section{Conclusion}

The present study shows that cue-target color similarity has a boosting effect on attentional capture by onset cues during search for onset-defined targets. A contingent-capture effect based on a match of the cues' onsets to the top-down control settings directed to target onsets was therefore overestimated in past studies that had used the classic contingent-capture protocol of Folk et al. (1992). Future studies should avoid confounding influences of color matches on top-down contingent-capture effects based on stimulus onsets, in order to provide an appropriate estimate of these effects. Furthermore, our results add to the growing body of evidence showing that topdown attentional control settings are more complex than hitherto has been thought, that they can comprise multiple features, and that the control setting depends on the task context.

Author note We thank Arnold Burghardt and Markus Grüner for their help with the data collection. Supported by Wiener Wissenschafts- und Technologiefonds Grant No. CS11-009 to U.A., Shelley Buchinger, and Otmar Scherzer.

\section{References}

Adamo, M., Pun, C., Pratt, J., \& Ferber, S. (2008). Your divided attention, please! The maintenance of multiple attentional control sets over distinct regions in space. Cognition, 107, 295-303. doi:10.1016/j. cognition.2007.07.003

Anderson, B. A., \& Folk, C. L. (2010). Variations in the magnitude of attentional capture: Testing a two-process model. Attention, Perception, \& Psychophysics, 72, 342-352. doi:10.3758/ APP.72.2.342

Ansorge, U., \& Heumann, M. (2003). Top-down contingencies in peripheral cuing: The roles of color and location. Journal of Experimental Psychology: Human Perception and Performance, 29, 947-958. doi:10.1037/0096-1523.29.5.937

Ansorge, U., \& Heumann, M. (2004). Peripheral cuing by abrupt-onset cues: The influence of color in S-R corresponding conditions. Acta Psychologica, 116, 115-143. doi:10.1016/j.actpsy.2004.01.001

Ansorge, U., \& Horstmann, G. (2007). Preemptive control of attentional capture by colour: Evidence from trial-by-trial analyses and orderings of onsets of capture effects in reaction time distributions. Quarterly Journal of Experimental Psychology, 60, 952-975. doi: 10.1080/17470210600822795

Ansorge, U., Kiss, M., Worschech, F., \& Eimer, M. (2011). The initial stage of visual selection is controlled by top-down task set: New ERP evidence. Attention, Perception, \& Psychophysics, 73, 113122. doi:10.3758/s13414-010-0008-3
Awh, E., Belopolsky, A. V., \& Theeuwes, J. (2012). Top-down versus bottom-up attentional control: A failed theoretical dichotomy. Trends in Cognitive Sciences, 16, 437-443. doi:10.1016/j.tics. 2012.06.010

Bacon, W. F., \& Egeth, H. E. (1994). Overriding stimulus-driven attentional capture. Perception \& Psychophysics, 55, 485-496. doi: 10.3758/BF03205306

Becker, S. I., Folk, C. L., \& Remington, R. W. (2010). The role of relational information in contingent capture. Journal of Experimental Psychology: Human Perception and Performance, 36, 1460-1476. doi: $10.1037 / \mathrm{a} 0020370$

Belopolsky, A. V., Schreij, D., \& Theeuwes, J. (2010). What is top-down about contingent capture? Attention, Perception, \& Psychophysics, 72, 326-341. doi:10.3758/APP.72.2.326

Brainard, D. H. (1997). The psychophysics toolbox. Spatial Vision, 10, 433-436. doi:10.1163/156856897X00357

Bundesen, C., Habekost, T., \& Kyllingsbæk, S. (2005). A neural theory of visual attention: Bridging cognition and neurophysiology. Psychological Review, 112, 291-328. doi:10.1037/ 0033-295X.112.2.291

Burnham, B. R. (2013). Using response time distributions to examine top-down influences on attentional capture. Attention, Perception, \& Psychophysics, 75, 257-277. doi:10.3758/ s13414-012-0396-7

Carmel, T., \& Lamy, D. (2014). The same-location cost is unrelated to attentional settings: An object-updating account. Journal of Experimental Psychology: Human Perception and Performance, 40, 1465-1478. doi:10.1037/a0036383

Eimer, M., \& Kiss, M. (2008). Involuntary capture is determined by task set: Evidence from event-related brain potentials. Journal of Cognitive Neuroscience, 20, 1423-1433. doi:10.1162/jocn.2008.20099

Folk, C. L., \& Remington, R. (1998). Selectivity in distraction by irrelevant featural singletons: Evidence for two forms of attentional capture. Journal of Experimental Psychology: Human Perception and Performance, 24, 847-858. doi:10.1037/0096-1523.24.3.847

Folk, C. L., \& Remington, R. (2010). A critical evaluation of the disengagement hypothesis. Acta Psychologica, 135, 103-105. doi: 10.1016/j.actpsy.2010.04.012

Folk, C. L., Remington, R. W., \& Johnston, J. C. (1992). Involuntary covert orienting is contingent on attentional control settings. Journal of Experimental Psychology: Human Perception and Performance, 18, 1030-1044. doi:10.1037/0096-1523.18.4. 1030

Fuchs, I., Theeuwes, J., \& Ansorge, U. (2013). Exogenous attentional capture by subliminal abrupt-onset cues: Evidence from contrastpolarity independent cueing effects. Journal of Experimental Psychology: Human Perception and Performance, 39, 974-988. doi:10.1037/a0030419

Goller, F., \& Ansorge, U. (2015). There is more to trial history than priming in attentional capture experiments. Attention, Perception, \& Psychophysics, 77, 1574-1584. doi:10.3758/ s13414-015-0896-3

Irons, J. L., Folk, C. L., \& Remington, R. W. (2012). All set! Evidence of simultaneous attentional control settings for multiple target colors. Journal of Experimental Psychology: Human Perception and Performance, 38, 758-775. doi:10.1037/a0026578

Kim, M. S., \& Cave, K. R. (1999). Top-down and bottom-up attentional control: On the nature of interference from a salient distractor. Perception \& Psychophysics, 61, 1009-1023. doi:10.3758/ BF03207609

Lamy, D. F., \& Kristjánsson, Á. (2013). Is goal-directed attentional guidance just intertrial priming? A review. Journal of Vision, 13(3), 14. doi:10.1167/13.3.14

Lamy, D., Leber, A., \& Egeth, H. E. (2004). Effects of task relevance and stimulus-driven salience in feature-search mode. Journal of 
Experimental Psychology: Human Perception and Performance, 30, 1019-1031. doi:10.1037/0096-1523.30.6.1019

Leber, A. B., \& Egeth, H. E. (2006). It's under control: Top-down search strategies can override attentional capture. Psychonomic Bulletin \& Review, 13, 132-138. doi:10.1037/0096-1523.30.6.1019

Liao, H.-I., \& Yeh, S.-L. (2013). Capturing attention is not that simple: Different mechanisms for stimulus-driven and contingent capture. Attention, Perception, \& Psychophysics, 75, 1703-1714. doi: 10.3758/s13414-013-0537-7

Ludwig, C. J., \& Gilchrist, I. D. (2002). Stimulus-driven and goal-driven control over visual selection. Journal of Experimental Psychology: Human Perception and Performance, 28, 902912. doi:10.1037/0096-1523.28.4.902

McDonald, J. J., Green, J. J., Jannati, A., \& Di Lollo, V. (2013). On the electrophysiological evidence for the capture of attention. Journal of Experimental Psychology: Human Perception and Performance, 39, 849-860. doi:10.1037/a0030510

Maljkovic, V., \& Nakayama, K. (1994). Priming of pop-out: I. Role of features. Memory \& Cognition, 22, 657-672. doi:10.3758/ BF03209251

Mast, F., \& Frings, C. (2014). The impact of the irrelevant: The task environment modulates the impact of irrelevant features in response selection. Journal of Experimental Psychology: Human Perception and Performance, 40, 2198-2213. doi:10.1037/a0038182

Pelli, D. G. (1997). The VideoToolbox software for visual psychophysics: Transforming numbers into movies. Spatial Vision, 10, 437-442. doi:10.1163/156856897X00366

Remington, R. W., Folk, C. W., \& McLean, J. P. (2001). Contingent attentional capture or delayed allocation of attention? Perception \& Psychophysics, 63, 298-307. doi:10.3758/BF03194470
Schreij, D., Los, S. A., Theeuwes, J., Enns, J. T., \& Olivers, C. N. L. (2014). The interaction between stimulus-driven and goal driven orienting as revealed by eye movements. Journal of Experimental Psychology: Human Perception and Performance, 40, 378-390. doi:10.1037/a0034574

Schreij, D., Theeuwes, J., \& Olivers, C. N. L. (2010). Abrupt onsets capture attention independent of top-down control settings II: Additivity is no evidence for filtering. Attention, Perception, \& Psychophysics, 72, 672-682. doi:10.3758/APP.72.3.672

Theeuwes, J. (2010). Top-down and bottom-up control of visual selection. Acta Psychologica, 135, 77-99. doi:10.1016/j.actpsy.2010.02.006

Theeuwes, J. (2013). Feature-based attention: It is all bottom-up priming. Philosophical Transactions of the Royal Society B, 368, 20130055. doi:10.1098/rstb.2013.0055

Theeuwes, J., Atchley, P., \& Kramer, A. F. (2000). On the time course of top-down and bottom-up control of visual attention. Control of cognitive processes: Attention and performance XVIII (pp. 105-124). Cambridge, MA: MIT Press.

Wolfe, J. M. (1994). Guided Search 2.0: A revised model of visual search. Psychonomic Bulletin \& Review, 1, 202-238. doi:10.3758/ BF03200774

Worschech, F., \& Ansorge, U. (2012). Top-down search for color prevents voluntary directing of attention to informative singleton cues. Experimental Psychology, 59, 153-162. doi:10.1027/1618-3169/ a000138

Wyble, B., Folk, C., \& Potter, M. C. (2013). Contingent attentional capture by conceptually relevant images. Journal of Experimental Psychology: Human Perception and Performance, 39, 861-871. doi: $10.1037 / \mathrm{a} 0030517$ 